\title{
An explanation for the different climate sensitivities of land and ocean surfaces based on the diurnal cycle
}

\author{
Axel Kleidon and Maik Renner \\ Biospheric Theory and Modelling Group, Max-Planck-Institut für Biogeochemie, Jena, Germany \\ Correspondence to: Axel Kleidon (akleidon@bgc-jena.mpg.de)
}

Received: 5 May 2017 - Discussion started: 19 May 2017

Revised: 23 August 2017 - Accepted: 27 August 2017 - Published: 25 September 2017

\begin{abstract}
Observations and climate model simulations consistently show a higher climate sensitivity of land surfaces compared to ocean surfaces. Here we show that this difference in temperature sensitivity can be explained by the different means by which the diurnal variation in solar radiation is buffered. While ocean surfaces buffer the diurnal variations by heat storage changes below the surface, land surfaces buffer it mostly by heat storage changes above the surface in the lower atmosphere that are reflected in the diurnal growth of a convective boundary layer. Storage changes below the surface allow the ocean surface-atmosphere system to maintain turbulent fluxes over day and night, while the land surface-atmosphere system maintains turbulent fluxes only during the daytime hours, when the surface is heated by absorption of solar radiation. This shorter duration of turbulent fluxes on land results in a greater sensitivity of the land surface-atmosphere system to changes in the greenhouse forcing because nighttime temperatures are shaped by radiative exchange only, which are more sensitive to changes in greenhouse forcing. We use a simple, analytic energy balance model of the surface-atmosphere system in which turbulent fluxes are constrained by the maximum power limit to estimate the effects of these different means to buffer the diurnal cycle on the resulting temperature sensitivities. The model predicts that land surfaces have a $50 \%$ greater climate sensitivity than ocean surfaces, and that the nighttime temperatures on land increase about twice as much as daytime temperatures because of the absence of turbulent fluxes at night. Both predictions compare very well with observations and CMIP5 climate model simulations. Hence, the greater climate sensitivity of land surfaces can be explained by its buffering of diurnal variations in solar radiation in the lower atmosphere.
\end{abstract}

\section{Introduction}

It has long been reported that the sensitivity of near-surface air temperatures over land is greater than over ocean, with land surfaces warming about $50 \%$ more strongly than ocean surfaces (Huntingford and Cox, 2000; Sutton et al., 2007; Boer, 2011; Byrne and O'Gorman, 2013). This phenomenon has also been found in observations, with the ratio remaining surprisingly constant through time (Lambert and Chiang, 2007). Several explanations have been put forth to explain this robust feature, including the role of heat transport (Boer, 2011), a balancing effect of oceanic heat storage (Lambert and Chiang, 2007), changes in evapotranspiration (Sutton et al., 2007) and the climatological relative humidity over land as well as its change (Byrne and O'Gorman,
2013). Also, Joshi and Gregory (2008) showed that this effect depends on the nature of the forcing, so that the ratio of land warming to ocean warming of about 1.5 holds only for changes in the greenhouse forcing.

Here, we explain this phenomenon of a higher climate sensitivity over land by the different ways of how the strong diurnal variation in solar radiation is buffered within the system (see Fig. 1). This buffering is accomplished by heat storage changes within the surface-atmosphere system that are forced by the heating by absorption of solar radiation during the day. The build-up of heat storage during the day then allows for nighttime temperatures that are far warmer than those one would expect in the absence of solar radiative heating at night. For ocean surfaces, these heat storage changes 
(a)

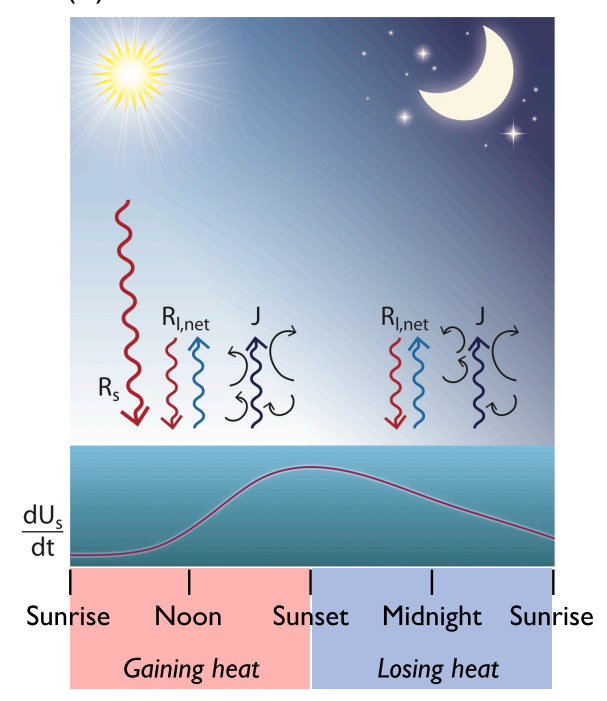

(b)

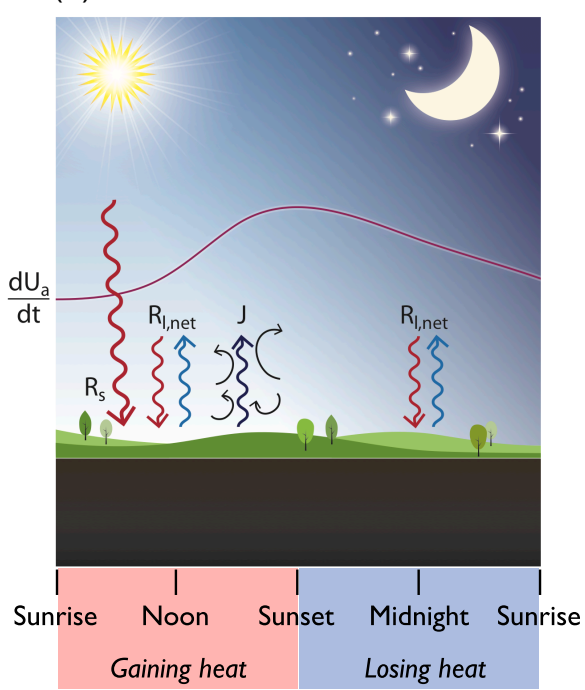

Figure 1. Schematic diagram of the surface energy balance of (a) an ocean surface and (b) the land surface. The main point to explain the different temperature sensitivity is related here to the different way by which these surfaces buffer the diurnal variation in solar radiation $\left(R_{\mathrm{S}}\right)$. The ocean surface buffers it by heat storage changes below the surface in the upper ocean (shown by the red line labeled $\mathrm{d} U_{\mathrm{s}} / \mathrm{d} t$ ), while the land surface buffers it primarily in the lower atmosphere (shown by $\mathrm{d} U_{\mathrm{a}} / \mathrm{d} t$ ). This results in stable conditions over land during nighttime, which prevent turbulent fluxes $(J)$ and which make surface temperature more sensitive to changes in the greenhouse effect. Graphics: Annett Boerner.

take place in the surface ocean. Since water is transparent, solar radiation penetrates the surface ocean to quite some depth before it is absorbed. Combined with the large heat capacity of water, this results in diurnal heat storage changes that take place below the ocean surface (sketched by the red line on the left of Fig. 1 and marked by $\left.\mathrm{d} U_{\mathrm{s}} / \mathrm{d} t\right)$. The build-up of heat storage during the day then maintains radiative cooling and turbulent heat fluxes during the night, resulting in little diurnal variations in surface temperature and turbulent fluxes. These characteristics of the ocean surface energy balance are very well observed and understood (see, e.g., textbooks by Oke, 1987, and Hartmann, 1994, and review paper by Kawai and Wada, 2007).

Over land this situation is quite different. Solar radiation is absorbed at the surface (or above in a canopy), but not below the surface. This is because land surfaces are not transparent as water, and because the heat conductivity of soils is generally so low that diurnal variations in surface heating do not penetrate more than $5-10 \mathrm{~cm}$ into the ground, resulting in a ground heat flux that is generally small. Even in desert regions or for bare ground with strong surface heating and no evaporative cooling, the ground heat flux does typically not exceed more than $100 \mathrm{~W} \mathrm{~m}^{-2}$, which is small compared to the maximum absorption of $800 \mathrm{~W} \mathrm{~m}^{-2}$ or more of solar radiation during the day (see, e.g., textbooks by Oke, 1987, and Hartmann, 1994, and syntheses by Bennett et al., 2008, and Purdy et al., 2016). We argue here that the strong diurnal variation in solar radiation is thus not buffered below but rather above the surface in the lower atmosphere. These changes in heat storage manifest themselves in the diurnal growth of the convective boundary layer. This buffering above the surface has an important consequence for the fluxes of the surface energy balance. Turbulent fluxes only take place when the surface is heated by solar radiation during the day, which causes the near-surface air to become unstable, while the nighttime is characterised by stable conditions near the surface as little heat can be drawn from the heat storage below the surface. This prevents turbulent fluxes from taking place at night. These consequences for turbulent fluxes over land surfaces are well observed (e.g. Oke, 1987; Hartmann, 1994). We suggest that because of this absence of turbulent fluxes at night the cooling at night is thus determined only by radiative exchange. Turbulent cooling of the surface takes place during half of the whole day, while during the other half it is cooled by radiative exchange. This difference in cooling terms should make nighttime temperatures more sensitive to changes in the greenhouse effect than daytime temperatures, a well-known phenomenon reported in observations (Easterling et al., 1997), and this should result in a greater climate sensitivity of land surfaces as well.

We demonstrate this explanation with an extremely simple yet physically based energy balance model in which we incorporate the effects of where heat storage changes take place. The model yields analytic expressions for the different climate sensitivity of land and ocean surfaces as well as the different sensitivity of nighttime and daytime temperatures. In the following, we first describe this model in Sect. 2. We then illustrate the climatological mean state in Sect. 3, derive 


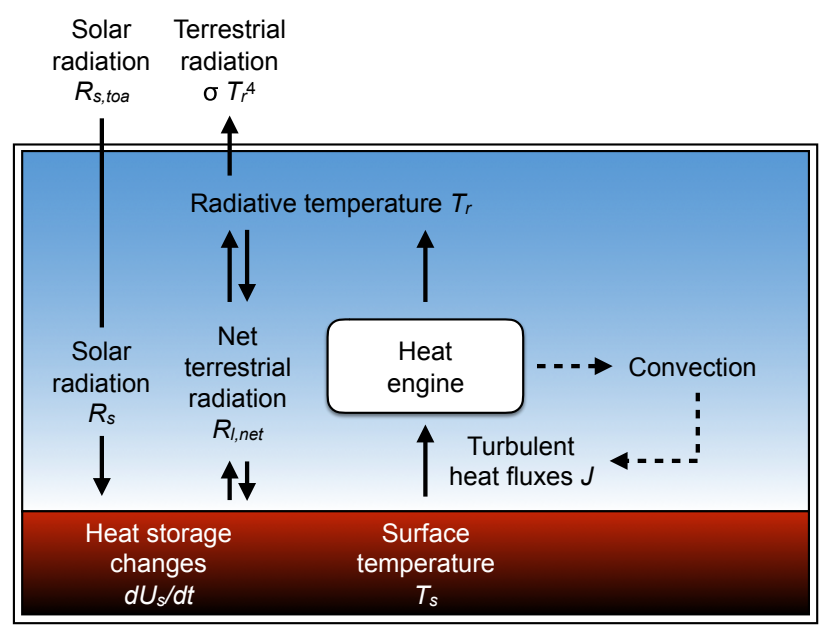

Figure 2. Schematic diagram of the surface energy balance used here in which turbulent heat fluxes are described as a result of an atmospheric heat engine operating between the surface and radiative temperatures. The limit to how much power can maximally be derived from this heat engine provides a means to parameterise turbulent heat fluxes.

analytic expressions for the ratios of these sensitivities, and compare these to CMIP5 climate model simulations. Some of the limitations of the model are then discussed, particularly regarding the description of terrestrial radiation and effects of the hydrologic cycle; our explanation is compared to previous interpretations of the difference in climate sensitivities; and we describe some of the implications and potentials for future research. We close with a brief summary and conclusions.

\section{Model description}

Our model consists of the energy balances of the surface and the whole surface-atmosphere system, a parameterisation of terrestrial radiation that is based on the grey atmosphere approximation, a formulation of turbulent fluxes derived from the thermodynamic constraint that these yield maximum power, and expressions for surface temperature that are derived from this model formulation. A schematic diagram of the model is provided in Fig. 2. The model formulation largely follows previous studies (Kleidon and Renner, 2013a, b; Kleidon et al., 2014, 2015). The main modifications here relate to the representation of heat storage changes and a formulation of terrestrial radiation based on the grey atmosphere approximation (as in Kleidon, 2016). The symbols used in the following description are summarised in Table 1.

\subsection{Energy balances}

For our description of the surface-atmosphere system, we need two basic energy balance constraints: the energy bal- ance of the surface and the energy balance of the whole system.

The surface energy balance is described in terms of heat storage changes that take place below the surface, $\mathrm{d} U_{\mathrm{s}} / \mathrm{d} t$; the absorbed solar radiation at the surface, $R_{\mathrm{s}}$; the net cooling by longwave radiation, $R_{1, \text { net }}$; and the turbulent heat fluxes, $J$ (the sum of the sensible and latent heat flux, which are combined here for simplicity):

$\frac{\mathrm{d} U_{\mathrm{s}}}{\mathrm{d} t}=R_{\mathrm{S}}-R_{\mathrm{l}, \text { net }}-J$.

The energy balance of the whole column is described by

$\frac{\mathrm{d} U_{\mathrm{tot}}}{\mathrm{d} t}=\frac{\mathrm{d} U_{\mathrm{s}}}{\mathrm{d} t}+\frac{\mathrm{d} U_{\mathrm{a}}}{\mathrm{d} t}=R_{\mathrm{s}, \text { toa }}-\sigma T_{\mathrm{r}}^{4}$,

where $\mathrm{d} U_{\text {tot }} / \mathrm{d} t$ represents the total change of heat storage within the surface-atmosphere system (consisting of heat storage changes below the surface, $\mathrm{d} U_{\mathrm{s}} / \mathrm{d} t$, and within the atmosphere, $\left.\mathrm{d} U_{\mathrm{a}} / \mathrm{d} t\right), R_{\mathrm{s}, \text { toa }}$ is the total absorption of solar radiation (surface and atmosphere) and $T_{\mathrm{r}}$ is the radiative temperature by which radiation is emitted to space, and $\sigma$ is the Stefan-Boltzmann constant. The radiative temperature is determined from the mean energy balance taken over a sufficiently long time so that

$T_{\mathrm{r}}=\left(\frac{R_{\mathrm{s}, \text { toa, avg }}}{\sigma}\right)^{1 / 4}$,

where $R_{\mathrm{S} \text {,toa, avg }}$ is the mean value of $R_{\mathrm{S}, \text { toa }}$. We assume that $T_{\mathrm{r}}$ does not change at the diurnal scale. This effectively represents our assumption that the system has sufficient capacity to store heat to balance out the variations in solar radiation.

The total change in heat storage within the system can be determined from the approximation that this total heat storage does not change when averaged over the course of day and night. The total change in heat storage can then be inferred from the difference between the instantaneous and mean solar forcing, given by

$\frac{\mathrm{d} U_{\text {tot }}}{\mathrm{d} t}=R_{\mathrm{s}, \text { toa }}-R_{\mathrm{s}, \text { toa,avg }}$.

In the following, we assume for simplicity that all solar radiation is absorbed at the surface, so that $R_{\mathrm{S}}=R_{\mathrm{S} \text {,toa }}$. This assumption simplifies the following considerations, but does not affect the results, as discussed in Sect. 4.1. We then describe the ocean-atmosphere system as a system in which the heat storage changes take place below the surface (that is, in the surface ocean), so that $\mathrm{d} U_{\mathrm{s}} / \mathrm{d} t=\mathrm{d} U_{\text {tot }} / \mathrm{d} t$. For the landatmosphere system, we neglect the ground heat flux, which is typically small on a diurnal timescale (as discussed in the introduction), so that the change in heat storage needs to take place in the lower atmosphere to meet this diurnal energy balance constraint. As it turns out, this heat storage change does not enter the formulations explicitly so that the term $\mathrm{d} U_{\mathrm{a}} / \mathrm{d} t$ does not appear in the equations below. 
Table 1. Variables and parameters used in this study.

\begin{tabular}{|c|c|c|c|}
\hline Symbol & Variable & Units (or value) & Usage \\
\hline G & Convective power & $\mathrm{W} \mathrm{m}^{-2}$ & Eq. (8) \\
\hline$J_{\mathrm{opt}}$ & Turbulent fluxes (optimised by max. power) & $\mathrm{W} \mathrm{m}^{-2}$ & Eq. (11) \\
\hline$k_{\mathrm{r}}$ & Radiative parameterisation constant & $\mathrm{W} \mathrm{m}^{-2} \mathrm{~K}^{-1}$ & Eq. (7) \\
\hline$R_{\mathrm{S}}$ & Surface absorption of solar radiation & $\mathrm{Wm}^{-2}$ & Sect. 2.1 \\
\hline$R_{1, \mathrm{~d}}$ & Downwelling flux of longwave radiation at the surface & $\mathrm{Wm}^{-2}$ & Sect. 2.2 \\
\hline$R_{1, \mathrm{u}}$ & Surface emission of longwave radiation & $\mathrm{Wm}^{-2}$ & Sect. 2.2 \\
\hline$R_{1, \text { net }}$ & Net flux of longwave radiation at the surface & $\mathrm{W} \mathrm{m}^{-2}$ & Eq. (5), Sect. 2.2 \\
\hline$R_{1, \text { net,opt }}$ & Net flux of longwave radiation at the surface (optimised by max. power) & $\mathrm{W} \mathrm{m}^{-2}$ & Eq. (11) \\
\hline$R_{1,0}$ & Radiative parameterisation constant & $\mathrm{W} \mathrm{m}^{-2}$ & Eq. (6) \\
\hline$T_{\mathrm{S}}$ & Surface temperature & $\mathrm{K}$ & Eq. (9) \\
\hline$T_{\text {day }}$ & Mean daytime temperature on land & $\mathrm{K}$ & Eq. (16) \\
\hline$T_{\text {land }}$ & Land surface temperature & $\mathrm{K}$ & Eq. (17) \\
\hline$T_{\text {night }}$ & Mean nighttime temperature on land & $\mathrm{K}$ & Eq. (15) \\
\hline$T_{\text {Ocean }}$ & Ocean surface temperature & $\mathrm{K}$ & Eq. (14) \\
\hline$T_{\text {global }}$ & Global mean surface temperature & $\mathrm{K}$ & Eq. (18) \\
\hline$\phi$ & Ratio of land to ocean warming & - & Eq. (21) \\
\hline$\tau$ & Longwave optical thickness & 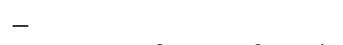 & Sect. 2.2 \\
\hline$\sigma$ & Stefan-Boltzmann constant & $5.67 \times 10^{-8} \mathrm{~W} \mathrm{~m}^{-2} \mathrm{~K}^{-4}$ & Eqs. (3) and (7) \\
\hline
\end{tabular}

\subsection{Parameterisation of longwave radiation}

To describe the net cooling by terrestrial radiation at the surface, $R_{1, \text { net }}$, we use a simple, linearised parameterisation of net longwave radiation of the form

$R_{\mathrm{l}, \mathrm{net}}=R_{\mathrm{l}, 0}+k_{\mathrm{r}}\left(T_{\mathrm{S}}-T_{\mathrm{r}}\right)$.

This formulation of net longwave radiation at the surface is similar to well-established empirical, linearised forms (e.g. Budyko, 1969), but it can also be derived from the grey atmosphere approximation of radiative transfer in combination with a linearisation of surface emission, as in Kleidon (2016). Net longwave radiation, $R_{1, \text { net }}$, is the difference between surface emission, $R_{\mathrm{l}, \mathrm{u}}=\sigma T_{\mathrm{s}}^{4} \approx T_{\mathrm{r}}+\left(4 \sigma T_{\mathrm{r}}^{3}\right)\left(T_{\mathrm{s}}-T_{\mathrm{r}}\right)$, and the downwelling flux of longwave radiation, $R_{1, \mathrm{~d}}=$ $(3 / 4) \tau \cdot R_{\mathrm{s}, \text { toa }}$. Here, $\tau$ is the longwave optical depth and $T_{\mathrm{r}}$ is described by Eq. (3). This interpretation has the advantage that the sensitivity of the parameters to a change in greenhouse forcing can directly be identified. The parameters $R_{1,0}$ and $k_{\mathrm{r}}$ are then related to the longwave optical depth, $\tau$; the mean emission of terrestrial radiation to space, $R_{\mathrm{s}, \text { toa, avg }}$; and the radiative temperature, $T_{\mathrm{r}}$, by

$R_{\mathrm{l}, 0}=\left(1-\frac{3}{4} \tau\right) \cdot R_{\mathrm{s}, \text { toa, avg }}$ and

$k_{\mathrm{r}}=4 \sigma T_{\mathrm{r}}^{3}=4 \cdot \frac{R_{\mathrm{s}, \mathrm{toa}, \mathrm{avg}}}{T_{\mathrm{r}}}$.

Note that a change in the greenhouse effect is associated with a change $\Delta \tau$, which alters the value of $R_{1,0}$, but not $k_{\mathrm{r}}$. A change in absorption of solar radiation, for instance due to enhanced reflectance by clouds or aerosols, affects both expressions if the total absorption, $R_{\mathrm{s}, \text { toa }}$, is altered.

\subsection{Turbulent fluxes determined from maximum power}

The turbulent fluxes $J$ in the surface energy balance are derived from the assumption that these operate at the thermodynamic limit of maximum power (Kleidon and Renner, 2013a). In this formulation, turbulent fluxes are seen as the driver of a convective, atmospheric heat engine that generates the power to sustain motion and the turbulent exchange between the surface and the atmosphere. This approach uses the common and well-accepted Carnot limit of a heat engine and combines it with the surface energy balance. The latter aspect plays a central role, because turbulent fluxes lower surface temperatures and thus affect the Carnot limit. The approach then assumes that natural systems evolve to and operate near their thermodynamic limit. This assumption 
falls into a broader range of thermodynamic optimality approaches. In particular, it relates closely to a general hypothesis of maximum entropy production (MEP; e.g. Ozawa et al., 2003; Martyushev and Seleznev, 2006; Kleidon et al., 2010; Kleidon, 2016), which has been applied rather successfully in the past to describe atmospheric dynamics (Paltridge, 1975; Ozawa and Ohmura, 1997; Lorenz et al., 2001; Kleidon et al., 2006) and to the mean climatological surface energy balance partitioning on land (Kleidon et al., 2014). As maximising the power of a heat engine results in maximum frictional dissipation, this is almost the same as maximising the associated entropy production of this process. The focus on maximising the power associated with turbulent heat fluxes, however, allows for a more specific application of thermodynamic optimality to the particular process of atmospheric turbulent heat transfer in relation to MEP, and it can be more easily explained using the well-established concept of a heat engine. In the following, this approach to estimate the magnitude of turbulent heat fluxes is briefly summarised and extended to include the effects of diurnal heat storage changes.

The power, $G$, or work per time, that a heat engine can provide is constrained by the Carnot limit, given by

$G=J \cdot \frac{T_{\mathrm{s}}-T_{\mathrm{r}}}{T_{\mathrm{s}}}$,

where for the application to vertical heat transfer in the atmosphere the driving temperature difference is set to the difference between the surface temperature and the radiative temperature. The motivation for using the radiative temperature as the cold temperature of the heat engine is to not use the temperature at a specific height of the atmosphere but rather to use the temperature at which the entropy export by radiative cooling to space is at a maximum. This temperature is, by definition, the radiative temperature, as it is the temperature of a blackbody that emits radiation at the rate $R_{\mathrm{s} \text {, toa, avg }}$.

To derive the maximum power limit from the Carnot limit, we combine this limit with a fundamental tradeoff by which a greater turbulent heat flux results in a lower surface temperature, so that the derived power has a maximum with an associated, optimum value of $J$ and $T_{\mathrm{s}}$. This tradeoff is obtained by combining the surface energy balance (Eq. 1) and the expression for $R_{1, \text { net }}$ (Eq. 5) to express $T_{\mathrm{S}}$ in terms of the radiative forcing, the heat storage change $\mathrm{d} U_{\mathrm{s}} / \mathrm{d} t$, and the turbulent fluxes $J$,

$T_{\mathrm{s}}=T_{\mathrm{r}}+\frac{R_{\mathrm{s}}-\mathrm{d} U_{\mathrm{s}} / \mathrm{d} t-R_{1,0}-J}{k_{\mathrm{r}}}$.

When used in the expression for the Carnot limit (Eq. 8), we obtain

$G=J \cdot \frac{R_{\mathrm{s}}-\mathrm{d} U_{\mathrm{s}} / \mathrm{d} t-R_{1,0}-J}{k_{\mathrm{r}} T_{\mathrm{s}}}$.

This expression has a maximum in power (i.e. maximum generation of turbulent kinetic energy), which can be derived analytically from $\mathrm{d} G / \mathrm{d} J=0$. When neglecting the variation in $T_{\mathrm{s}}$ with $J$ in the denominator, the maximisation yields an optimum heat flux, $J_{\text {opt }}$, and net longwave flux, $R_{1 \text {,net,opt }}$, given by

$$
\begin{aligned}
J_{\mathrm{opt}} & =\frac{R_{\mathrm{s}}-\mathrm{d} U_{\mathrm{s}} / \mathrm{d} t-R_{1,0}}{2} \\
R_{1, \text { net,opt }} & =\frac{R_{\mathrm{s}}-\mathrm{d} U_{\mathrm{s}} / \mathrm{d} t+R_{1,0}}{2} .
\end{aligned}
$$

Note how this formulation of surface energy balance partitioning depends on heat storage changes below the surface, $\mathrm{d} U_{\mathrm{s}} / \mathrm{d} t$, but not on heat storage changes that take place in the lower atmosphere, $\mathrm{d} U_{\mathrm{a}} / \mathrm{d} t$. We use these two contrasting cases of heat storage change to describe how this partitioning looks for ocean (day and night) and land surfaces (daytime only).

For the ocean surface, the dominant heat storage changes take place below the surface, so that $\mathrm{d} U_{\mathrm{s}} / \mathrm{d} t \approx \mathrm{d} U_{\text {tot }} / \mathrm{d} t=$ $R_{\mathrm{s} \text {,toa }}-R_{\mathrm{S} \text {, toa, avg }}$ (see Eq. 4). With this expression for $\mathrm{d} U_{\mathrm{s}} / \mathrm{d} t$, the optimum surface energy partitioning is then given by

$$
\begin{aligned}
J_{\text {opt }, \text { ocean }} & =\frac{R_{\mathrm{s}, \text { avg }}-R_{1,0}}{2} \\
R_{1, \text { net,opt,ocean }} & =\frac{R_{\mathrm{s}, \text { avg }}+R_{1,0}}{2} .
\end{aligned}
$$

This partitioning describes no temporal changes during the course of the day, as the turbulent fluxes as well as net longwave radiation are described by the mean solar radiation at the surface, $R_{\mathrm{s} \text {,avg }}$, rather than the instantaneous forcing, $R_{\mathrm{S}}$.

For the land surface, we assume that the heat storage changes take place in the lower atmosphere and $\mathrm{d} U_{\mathrm{s}} / \mathrm{d} t \approx 0$. Then, the energy balance partitioning during the day is given by

$$
\begin{aligned}
J_{\mathrm{opt}, \text { land }} & =\frac{R_{\mathrm{s}}-R_{1,0}}{2} \\
R_{1, \text { net, opt,land }} & =\frac{R_{\mathrm{s}}+R_{1,0}}{2} .
\end{aligned}
$$

Note how this partitioning includes the instantaneous rate of absorption of solar radiation, $R_{\mathrm{s}}$, thus resulting in a pronounced diurnal variation in surface energy balance partitioning as it is commonly observed on land. During night where $R_{\mathrm{S}}=0$ and $J=0$ due to the prevalent stable conditions, we assume $R_{1, \text { net }} \approx 0$. This simplification is reasonable as observations typically show that the cooling of the surface by net longwave radiation at night is less than $100 \mathrm{~W} \mathrm{~m}^{-2}$ (e.g. Oke, 1987; Hartmann, 1994) and thus much smaller than the peak heating rate by solar radiation during the day.

\subsection{Surface temperatures}

For the two contrasting cases of land and ocean surfaces, we can derive expressions for surface temperature by equating the optimum expressions for the net longwave radiative flux with Eq. (5). 
For the ocean surface, surface energy balance partitioning does not change over the course of day and night. Hence, surface temperature is constant and depends only on the mean absorption of solar radiation:

$T_{\text {ocean }}=T_{\mathrm{r}}+\frac{R_{\mathrm{s}, \mathrm{avg}}-R_{\mathrm{l}, 0}}{2 k_{\mathrm{r}}}$.

For land, we split the surface energy balance partitioning into two parts of night and day. The nighttime temperature is derived directly from $R_{1, \text { net }} \approx 0$. This yields an equation for the nighttime temperature of

$T_{\text {night }}=T_{\mathrm{r}}-\frac{R_{1,0}}{k_{\mathrm{r}}}$.

During the day, the mean absorption of solar radiation is about $R_{\mathrm{S}, \text { day }}=2 \cdot R_{\mathrm{s}, \text { avg }}$, which we use for $R_{\mathrm{S}}$ in Eq. (13). The mean daytime surface temperature is then given by

$T_{\text {day }}=T_{\mathrm{r}}+\frac{R_{\mathrm{s}, \text { day }}-R_{\mathrm{l}, 0}}{2 k_{\mathrm{r}}}$.

This yields a mean surface temperature over land, $T_{\mathrm{land}}=$ $1 / 2 \cdot\left(T_{\text {night }}+T_{\text {day }}\right)$, of

$T_{\text {land }}=T_{\mathrm{r}}+\frac{R_{\mathrm{S}, \text { avg }}-3 / 2 \cdot R_{\mathrm{l}, 0}}{2 k_{\mathrm{r}}}$

When both temperatures are combined, the global mean surface temperature, $T_{\text {global }}$, is described by

$T_{\text {global }}=\left(1-f_{\text {land }}\right) \cdot T_{\text {ocean }}+f_{\text {land }} \cdot T_{\text {land }}$,

where $f_{\text {land }}=0.29$ is the fraction of land area of the total surface area of the Earth.

Equations (14)-(18) represent the key equations used in the following to evaluate the sensitivity of surface temperature to a change in radiative forcing. These estimates are then compared to the respective sensitivities derived from the CMIP5 climate model simulations (Taylor et al., 2012), using the $4 \times \mathrm{CO}_{2}$ and preindustrial control simulations (a list of models used is provided in Table A1).

\section{Results and discussion}

\subsection{Global energy balance}

We first evaluate the energy balance partitioning and expressions for temperatures using global means. The forcing is described by observations of the total mean absorption of solar radiation of the surface-atmosphere system of about $R_{\mathrm{S}, \text { toa, avg }}=240 \mathrm{~W} \mathrm{~m}^{-2}$ and the mean absorption at the surface of $R_{\mathrm{S} \text {, avg }}=165 \mathrm{~W} \mathrm{~m}^{-2}$ (Stephens et al., 2012). To obtain a global mean surface temperature of about $288 \mathrm{~K}$, we choose a value of $\tau=1.74$ for the longwave optical depth. This is the only parameter in the formulations that we adjusted to match observations. From this radiative forcing, the parameters $R_{1,0}$ and $k_{\mathrm{r}}$ are derived for the surface energy balance partitioning. The resulting surface energy balance partitioning is illustrated in Fig. 3 and the respective values are provided in Table 2. The turbulent fluxes of $119 \mathrm{~W} \mathrm{~m}^{-2}$ and net longwave radiation of $46 \mathrm{~W} \mathrm{~m}^{-2}$ derived from the maximum power limit compare reasonably well to the estimates from observations of 112 and $53 \mathrm{~W} \mathrm{~m}^{-2}$ (Stephens et al., 2012). Note that the radiative properties as well as continental area show strong geographic variations that are not accounted for here, so that this evaluation merely shows the plausibility of the formulations.

The difference in diurnal energy balance partitioning between the ocean and land surface is illustrated for global mean conditions in Fig. 4. Note that these global mean conditions are hypothetical and used here to illustrate the difference in energy balance partitioning using the formulations described in the methods section. At the top of Fig. 4, the diurnal variation in the total heat storage within the surfaceatmosphere system is shown (compare with Eq. 4) for a mean absorption of solar radiation at the surface of $165 \mathrm{~W} \mathrm{~m}^{-2}$ and the respective diurnal variation in $R_{\mathrm{s}}$. The differences in energy balance partitioning for ocean and land surfaces, using Eqs. (12) and (13), are then illustrated in Fig. 4b and c. This figure clearly illustrates that by buffering the diurnal variations in solar radiation below the surface, the ocean surface energy balance has no diurnal variation in turbulent fluxes. If the buffering takes place above the surface, as is mostly the case for land surfaces, this results in a pronounced diurnal variation in turbulent fluxes.

\subsection{Temperature sensitivity to greenhouse forcing}

We next evaluate the case of global warming. An increase in the greenhouse effect is represented in our formulation by an increase in the longwave optical depth, $\Delta \tau>0$. We used a value of $\Delta \tau=0.18$ to obtain a global temperature increase of $\Delta T_{\text {global }}=5.0 \mathrm{~K}$. The increase in optical thickness then changes $R_{1,0}$ by $\Delta R_{1,0}=-3 / 4 \cdot R_{\mathrm{s}, \text { toa }} \cdot \Delta \tau<0$. Using the grey atmosphere approximation, this change in $\tau$ implies an increase in the downwelling longwave radiation of about $\Delta R_{1, \mathrm{~d}}=33 \mathrm{~W} \mathrm{~m}^{-2}$. This increase compares fairly well to the range found in CMIP5 simulations used here, which range from 20 to $42 \mathrm{~W} \mathrm{~m}^{-2}$ (global mean) and are associated with a warming of 2.9 to $6.0 \mathrm{~K}$ in surface temperatures $\left(4 \times \mathrm{CO}_{2}\right.$ scenario - PI control; see Fig. A1). The effect of this change in optical thickness on the diurnal course of surface energy balance partitioning is shown in Fig. $4 \mathrm{~b}$ and $\mathrm{c}$ by the dotted lines. The consequences for mean ocean and land temperatures as well as for daytime and nighttime temperatures on land is illustrated in Fig. 5, with values given in Table 2. These sensitivities can be derived analytically, using the expressions derived in Sect. 2.4. 


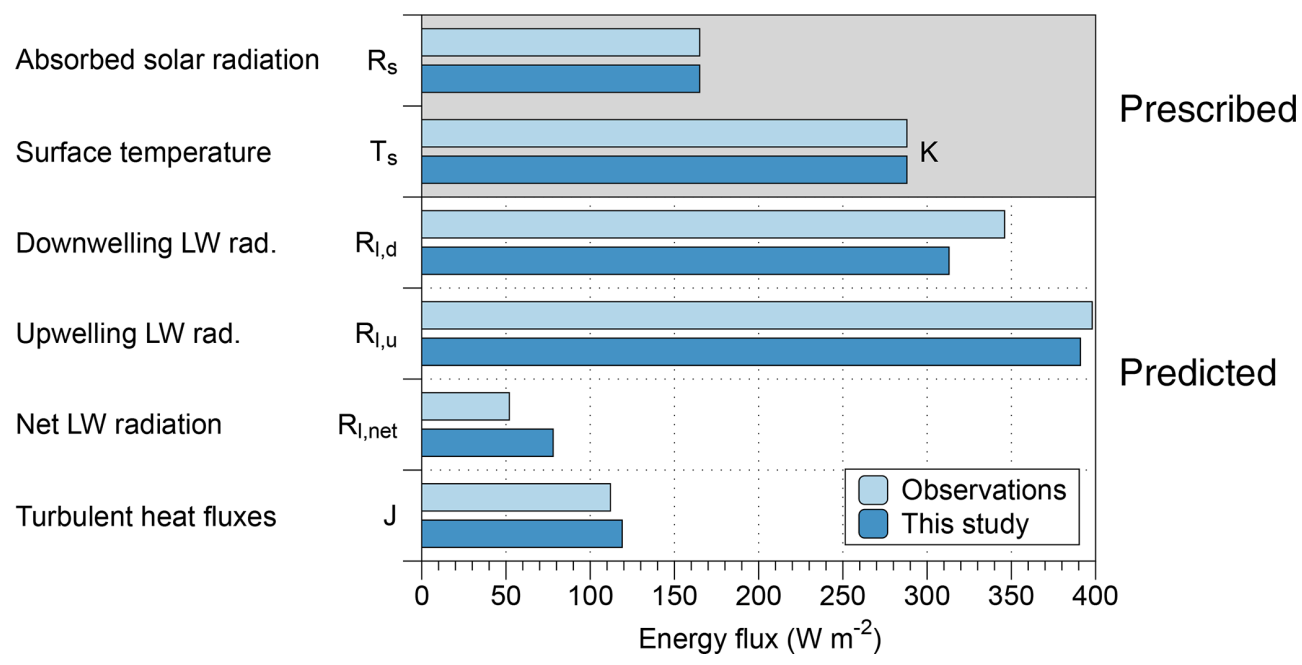

Figure 3. Global mean surface energy balance partitioning predicted from the approach used in this study in comparison to observations of Stephens et al. (2012). The respective values are provided in Table 2.

Table 2. Estimates for the global mean forcing, a global warming for a $4 \times \mathrm{CO}_{2}$ scenario, and a scenario of solar brightening.

\begin{tabular}{|c|c|c|c|c|c|}
\hline Symbol & Present day & Global warming & Difference & Solar brightening & Difference \\
\hline \multicolumn{6}{|l|}{ Forcing } \\
\hline$R_{\mathrm{S}, \text { toa, avg }}\left(\mathrm{W} \mathrm{m}^{-2}\right)$ & 240 & 240 & 0 & 240 & 0 \\
\hline$R_{\mathrm{S}, \text { avg }}\left(\mathrm{W} \mathrm{m}^{-2}\right)$ & 165 & 165 & 0 & 175 & 10 \\
\hline$\tau(-)$ & 1.74 & 1.924 & 0.18 & 1.74 & 0 \\
\hline \multicolumn{6}{|c|}{ Derived radiation properties } \\
\hline$T_{\mathrm{r}}(\mathrm{K})$ & 255 & 255 & 0 & 255 & 0 \\
\hline$R_{1,0}\left(\mathrm{~W} \mathrm{~m}^{-2}\right)$ & -73.2 & -106.3 & -33.1 & -73.2 & 0 \\
\hline$k_{\mathrm{r}}\left(\mathrm{W} \mathrm{m} \mathrm{m}^{-2} \mathrm{~K}^{-1}\right)$ & 3.76 & 3.76 & 0 & 3.76 & 0 \\
\hline \multicolumn{6}{|c|}{ Predicted surface energy balance } \\
\hline$J_{\mathrm{opt}}\left(\mathrm{W} \mathrm{m}^{-2}\right)$ & 119 & 136 & 16.6 & 124 & 5.0 \\
\hline$R_{1, \text { net,opt }}\left(\mathrm{W} \mathrm{m}^{-2}\right)$ & 46 & 29 & -16.6 & 51 & 5.0 \\
\hline \multicolumn{6}{|c|}{ Predicted temperatures } \\
\hline$T_{\text {ocean }}(\mathrm{K})$ & 286.7 & 291.1 & 4.4 & 288.0 & 1.3 \\
\hline$T_{\text {land }}(\mathrm{K})$ & 291.6 & 298.2 & 6.6 & 292.9 & 1.3 \\
\hline$T_{\text {global }}(\mathrm{K})$ & 288.1 & 293.2 & 5.0 & 289.5 & 1.3 \\
\hline
\end{tabular}

The warming of the ocean surface, $\Delta T_{\text {ocean, }}$ is then given by

$\Delta T_{\text {ocean }}=-\frac{\Delta R_{1,0}}{2 k_{\mathrm{r}}}$.

When the same change of optical thickness is applied to land, it results in a warming of the land surface, $\Delta T_{\text {land }}$, of

$\Delta T_{\text {land }}=-\frac{3}{2} \frac{\Delta R_{1,0}}{2 k_{\mathrm{r}}}$.
When taking the ratio, $\phi$, of these two changes, we obtain

$\phi=\frac{\Delta T_{\text {land }}}{\Delta T_{\text {ocean }}}=\frac{3}{2}$

Hence, the land surface is $50 \%$ more sensitive to a change in longwave optical depth than the ocean surface. We can also translate this fixed ratio between land and ocean warming into respective expressions that relate land and ocean warm- 
(a) Diurnal heat storage variations

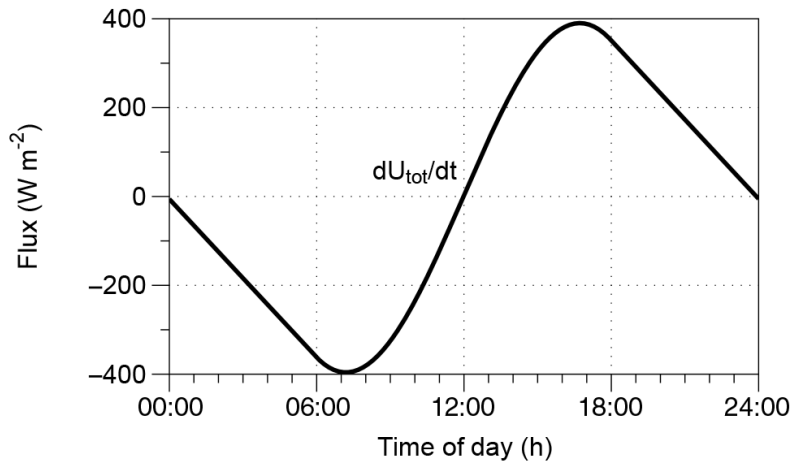

(b) Ocean surface

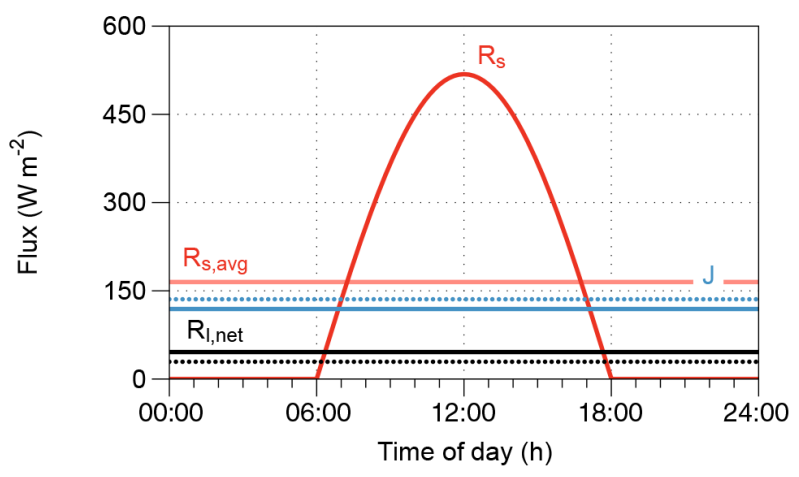

(c) Land surface

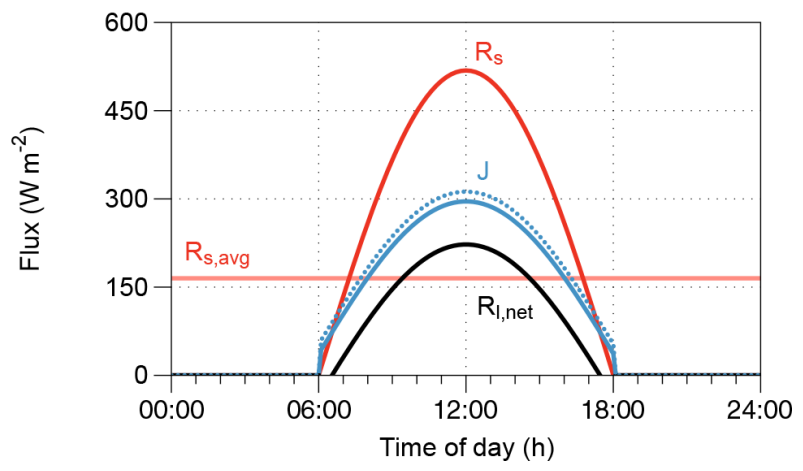

Figure 4. Mean diurnal heat storage variations inferred from the solar forcing (a) and the associated diurnal variations in surface energy balance partitioning of (b) an ocean surface and (c) a land surface as predicted by the approach described here. The pale red line in panels (b) and (c) refers to the average of absorbed solar radiation at the surface of $R_{\mathrm{s}, \text { avg }}=165 \mathrm{~W} \mathrm{~m}^{-2}$. The dotted lines in panels (b) and (c) refer to the respective values for a global mean warming of $\Delta T_{\mathrm{S}}=5 \mathrm{~K}$ due to changes in greenhouse forcing.

ing to the global temperature change:

$$
\begin{aligned}
\Delta T_{\text {ocean }} & =\frac{1}{1+0.5 \cdot f_{\text {land }}} \cdot \Delta T_{\text {global }} \approx 0.87 \cdot \Delta T_{\text {global }} \\
\Delta T_{\text {land }} & =\frac{3}{2-f_{\text {land }}} \cdot \Delta T_{\text {global }} \approx 1.31 \cdot \Delta T_{\text {global }} .
\end{aligned}
$$

When using the global mean values as shown in Table 2, a global mean temperature increase of $5 \mathrm{~K}$ due to an increased

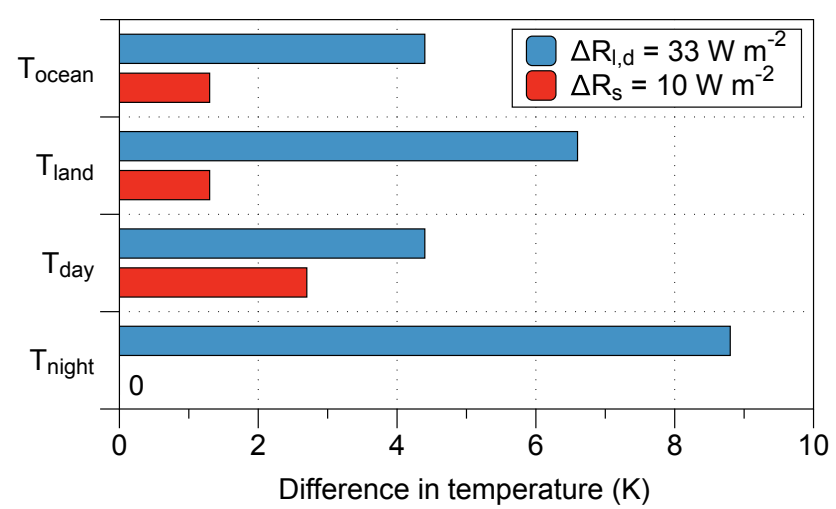

Figure 5. Difference in ocean and land temperatures as well as daytime and nighttime temperatures resulting from an enhanced greenhouse effect $\left(\Delta R_{1, \mathrm{~d}}=+33 \mathrm{~W} \mathrm{~m}^{-2}\right)$ and from enhanced absorption of solar radiation at the surface $\left(\Delta R_{\mathrm{S}}=+10 \mathrm{~W} \mathrm{~m}^{-2}\right)$, using the values from Table 2 .

greenhouse effect translates into an increase by $6.6 \mathrm{~K}$ over land (or $31 \%$ more than the global mean increase), while oceans only increase by $4.4 \mathrm{~K}$ (or $13 \%$ less than the global mean increase). In Fig. 6 we compare the predicted ratio $\phi$ as well as the temperature differences $\Delta T_{\text {land }}$ and $\Delta T_{\text {ocean }}$ to $\Delta T_{\text {global }}$ to the respective simulated values of CMIP5 climate model simulations. Although some deviations can be seen, our estimates overall compare very well to the global mean changes found in the CMIP5 simulations.

As argued in the introduction, the difference in the climate sensitivity of land and ocean surfaces should be attributable to the different behaviour of the land surface at night than during the day. To evaluate this in our formulations, we also looked at the sensitivities of nighttime and daytime temperatures as proxies for minimum and maximum temperatures. The minimum temperature typically occurs at the end of the night, and we approximate it by the use of $T_{\text {night }}$. The maximum temperature occurs at the end of the day, and for this temperature we use $T_{\text {day }}$. Using the above expressions for these temperatures, we obtain

$$
\begin{aligned}
\Delta T_{\text {night }} & =-\frac{\Delta R_{1,0}}{k_{\mathrm{r}}} \\
\Delta T_{\text {day }} & =-\frac{\Delta R_{\mathrm{l}, 0}}{2 k_{\mathrm{r}}}=\frac{1}{2} \Delta T_{\text {night }} .
\end{aligned}
$$

Hence, minimum temperatures increase at twice the rate of maximum temperatures in our formulation, thus reducing the diurnal temperature range. This is broadly consistent with observations, for which a range of about 1.6-2.4 is reported for most seasons (Horton, 1995; Easterling et al., 1997), although in observations the ratio varies between hemispheres and seasons.

We did not perform an evaluation of the diurnal temperature range for the CMIP5 simulations for a few reasons. There are other effects, e.g. due to changes in the hydrologic cycle, as well as model biases that quite substantially affect 


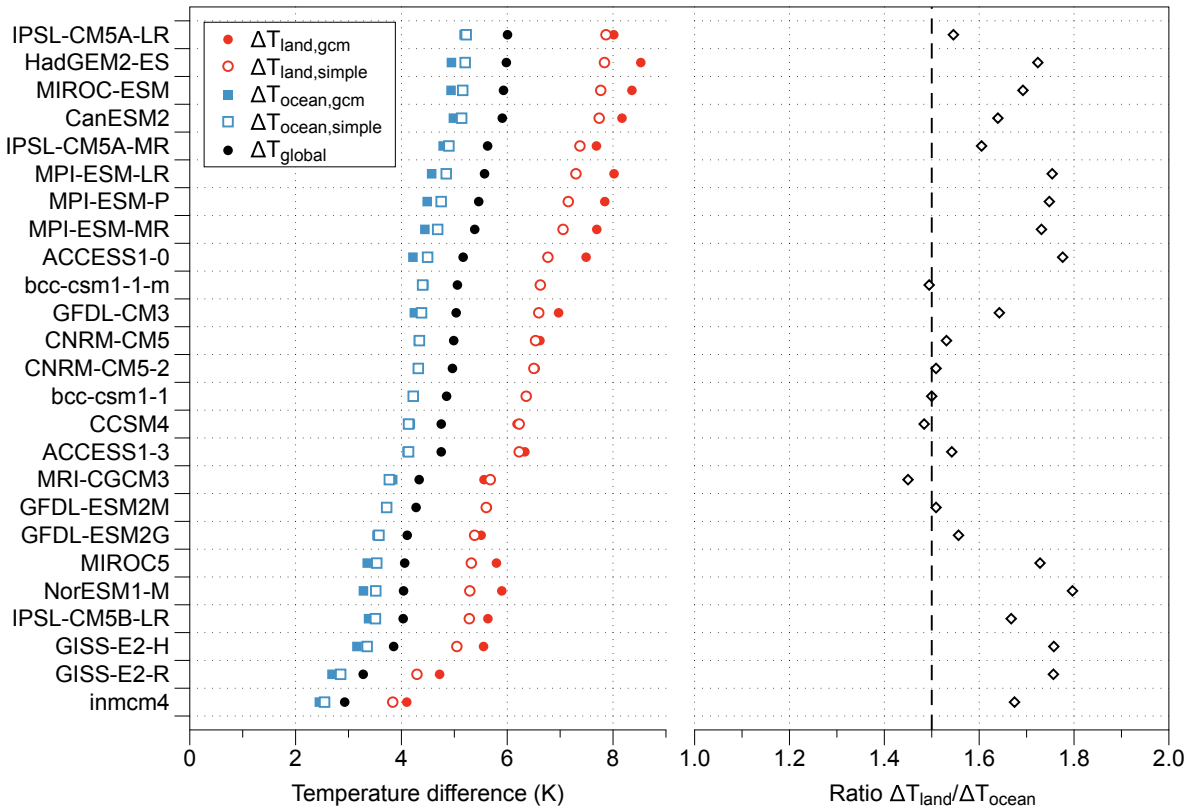

Figure 6. Global mean response of 25 CMIP5 climate model simulations. Shown is the mean warming of the ocean surface $\left(\Delta T_{\mathrm{Ocean}, \mathrm{gcm}}\right.$, blue solid squares), the land surface ( $\Delta T_{\text {land,gcm }}$, red solid circles), and the global mean $\left(\Delta T_{\text {global }}\right.$, black dots) between the $4 \times \mathrm{CO}_{2}$ and

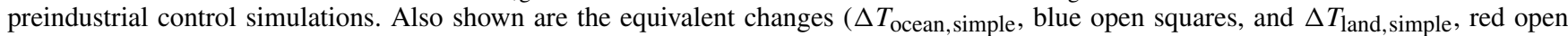
circles) predicted from the energy balance considerations made here. The right diagram directly compares the ratio $\phi=\Delta T_{\text {land }} / \Delta T_{\mathrm{ocean}}$ from the GCM simulations (diamonds) to the prediction made here (dashed line).

the trend in the diurnal temperature range in the CMIP5 simulations (Lindvall and Svensson, 2015) so that this direct effect of an enhanced greenhouse forcing is not the dominant factor in the $4 \times \mathrm{CO}_{2}$ simulations. These effects would need to be accounted for in our expressions before a more detailed comparison could be made. Yet, the well-established observation that the diurnal temperature range decreases with global warming is consistent with our interpretation why the climate sensitivity of land is higher than for ocean surfaces.

\subsection{Temperature sensitivity to solar forcing}

To illustrate that changes in solar radiation affect the temperature sensitivity quite differently, as described in Joshi and Gregory (2008), we consider a case in which the total absorption of solar radiation is unchanged, but more solar radiation is absorbed at the surface (i.e. $\Delta R_{\mathrm{s}, \mathrm{avg}}=+10 \mathrm{~W} \mathrm{~m}^{-2}$ ). This magnitude of change in solar absorption is comparable to observed changes associated with solar dimming and brightening over the last decades (e.g. Wild, 2009), which in turn relates mostly to changes in aerosol concentrations in the atmosphere. For comparability, we use the same value for the longwave optical depth and considered the case of solar brightening to better compare the effects of solar changes to changes in greenhouse forcing. The sensitivity to absorbed solar radiation at the surface is shown in Fig. 5 by the red bars, with the values given in Table 2 in the column labelled "Solar Brightening".
Our simple estimates partition the increase in surface solar absorption equally into increases in $\Delta J$ and $\Delta R_{1, \text { net }}$ (see Eqs. 12 and 13). The change in ocean and land temperatures is given by (see Eqs. 14 and 17)

$\Delta T_{\text {ocean }}=\frac{\Delta R_{\mathrm{s}, \mathrm{avg}}}{2 k_{\mathrm{r}}}=\Delta T_{\text {land }}$,

and the land surface warms on average by the same amount as the ocean surface. This is quite different than the result from the change in the greenhouse effect, where the sensitivities were different. The effect on the diurnal temperature range on land is also markedly different. While the nighttime temperatures remain unchanged as they do not depend on $R_{\mathrm{S}}$, the daytime temperatures are increased by twice the mean warming, with $\Delta T_{\text {day }}=\Delta R_{\mathrm{s}} / k_{\mathrm{r}}=2 \Delta T_{\text {land }}$. This effect of solar radiation on maximum temperatures is well known (e.g. Wild, 2009) and has been used to infer solar radiation from the diurnal temperature range (e.g. Bristow and Campbell, 1984).

Our formulations thus show that the temperature sensitivities of ocean and land surfaces, as well as sensitivities of minimum and maximum temperatures on land, and thus of the diurnal temperature range, are closely connected and react differently depending on the type of radiative change at the surface. 


\section{Discussion}

\subsection{Limitations}

Despite its physical basis, our model has, obviously, several potential limitations due to its extremely simple nature. These potential limitations relate to the parameterisation of radiation and turbulent fluxes, as well as to how evaporation is treated in our formulations.

To start, the use of the grey atmosphere approximation for the downwelling flux of longwave radiation is an approximation. It represents a more mechanistic approach of parameterising longwave radiative transfer, with the main difference to earlier work (Kleidon and Renner, 2013a, b) being the additive constant $R_{1,0}$ in Eq. (5) that played here an important role. The use of the grey atmosphere approximation, however, is likely to overestimate the downward longwave flux for a given optical depth. Turbulent fluxes cause a lower surface temperature than in radiative equilibrium, which results in a colder lower atmosphere that is in radiative-convective equilibrium. This, in turn, should be associated with a lower downwelling flux of longwave radiation. By using the grey atmosphere approximation, we do not account for this effect, which is likely to result in some biases in our formulation. This is likely to result in an overestimation of the sensitivity of surface temperature to changes in the optical depth. However, as we do not calculate optical depths or use observations but rather adjust it to represent the global mean temperature or a given temperature change, the effect of this bias in the radiation parameterisation is likely to be small for our results.

We also did not specifically consider absorption of solar radiation within the atmosphere (which can be seen by comparing $R_{\mathrm{s} \text {, toa,avg }}$ to $R_{\mathrm{s} \text {,avg }}$ in Table 2). This atmospheric absorption would result in some diurnal variation in heat storage within the atmosphere over oceans. However, since our expressions do not explicitly depend on changes in atmospheric heat storage, the effect of this should not change our results.

Another limitation relates to our representation of turbulent fluxes. We used the Carnot limit and the assumption that turbulent fluxes operate near the limit of maximised power. Yet, the diurnal variation in heat storage in the lower atmosphere over land may need to be accounted for in the derivation of thermodynamic limits, which may then result in a different partitioning of energy fluxes at the surface. However, as long as the turbulent fluxes on land are proportional to the instantaneous value of absorbed solar radiation at the surface (which is a good assumption as turbulent fluxes on land show a strong diurnal variation), turbulent fluxes must then be small at night. The basic reasoning would then still apply that nighttime temperatures are more sensitive to a change in greenhouse forcing, thus resulting in an altered climate sensitivity of land surfaces compared to ocean surfaces, although the specific ratio $\phi$ may differ from our value of $3 / 2$.
Furthermore, we do not explicitly consider evaporation in our formulation, but include it in the turbulent fluxes $J$. Evaporation and the associated latent heat flux cools the surface, yet it only heats the atmosphere (and the surface) when it condenses. At the global scale in steady state, evaporation needs to balance precipitation, so that evaporation does not necessarily need to be represented as a separate term in the surface energy balance. Yet, spatiotemporal imbalances between evaporation and precipitation due to storage changes of water vapour and moisture transport can result in regional temperature variations due to evaporation (Kleidon and Renner, 2013a). Furthermore, differences in radiative parameters during dry and wet periods may result in further modulations of surface temperatures that we did not account for and that could have an effect (Rochetin et al., 2014). Those effects would clearly need to be addressed when our formulations are applied to the regional scale, which could form a topic of future research.

Yet, overall, it would seem that despite these deficiencies, our simple representation is able to adequately illustrate our explanation from the introduction in a parsimonious way as it captures the difference in climate sensitivity of ocean and land surfaces, and connects this difference to the difference in sensitivity between minimum and maximum temperatures.

\subsection{Interpretation}

Our explanation for the difference in temperature sensitivity between oceans and land is quite different, yet not in contradiction to previous approaches, which we explain in the following. Previous attempts to explain the difference in temperature sensitivity typically start with the reduced water availability on land. In arid regions, water limits evaporation, so that the radiative heating results in an enhanced sensible heat flux and thermal emission, which is accomplished by a warmer surface temperature. When ocean and land are exposed to an equal increase in the downwelling flux of longwave radiation by an enhanced greenhouse effect, the land surface in arid regions should respond by a stronger warming than the ocean surface (which to some extent is found in climate model simulations). These effects then result in different levels of humidity and affect lapse rates in the lower atmosphere. This line of explanation was developed and extended by Sutton et al. (2007), Joshi et al. (2008) and Byrne and O'Gorman (2013).

Our approach uses a systems approach to the surfaceatmosphere system, so it neither focuses entirely on the surface energy balance nor on the atmosphere, but rather on the coupled system. This system is subjected to the energy balance constraint during the diurnal cycle, which we use to infer the buffering needed to level out the strong variation in solar radiation. While the buffering below the ocean surface is relatively straightforward and established for the oceanatmosphere system, the buffering in the lower atmosphere for the land-atmosphere system is less established but cen- 
tral to the explanation presented here. This buffering implies the formation of a convective boundary layer on land during the day to heat the lower atmosphere and to accomplish the diurnal heat storage change. It also implies unstable conditions during the day that drives the sensible heat flux, dry convection, and boundary layer development. This likely results in a greater lapse rate that is closer to the dry adiabatic lapse rate as the heating of the lower atmosphere is primarily driven by the sensible heat flux and dry convection. These consequences link to the properties that were used before to explain the difference in temperature sensitivity, so our explanation is consistent with previous interpretations in this respect. Yet, what our approach shows is that these dynamics do not need to be resolved in detail to derive the difference in climate sensitivity, as these essentially follow from the energy balance closure assumption applied to the surfaceatmosphere system over the whole day. It would thus seem that this energy balance closure and the way by which the land surface-atmosphere system accomplishes it are the primary cause for the difference in temperature sensitivity.

Our explanation can not only explain the difference in temperature sensitivity over land and ocean but also connects to the well-established difference in sensitivity of minimum and maximum temperatures as well as the distinctively different diurnal course of turbulent fluxes between land and ocean. In this sense, our explanation appears to be more general, as it is able to explain more phenomena by a less complex approach.

\subsection{Further implications and potential for future research}

We can draw a few broader implications from these insights that open up possibilities for a range of further research.

First, our results explain why the diurnal dynamics of the surface energy balance of ocean and land surfaces are so distinctively different. While these differences are well established in observations and are described in textbooks (e.g. Oke, 1987; Hartmann, 1994), we can explain these by the different means by which the diurnal variation in solar radiation is being buffered, with critical implications for the temperature sensitivity. For the ocean, variations in solar radiation are buffered by heat storage changes below the surface, so that turbulent fluxes do not show much of a diurnal variation. On land, however, it is well known that turbulent fluxes show a pronounced diurnal variation during the day and are practically absent during the night. We interpreted this different behaviour of land surfaces here as a result of the buffering taking place in the lower atmosphere above the surface, rather than below as in the case for the ocean, which results in stable conditions during nighttime that are more sensitive to changes in the greenhouse effect. As variations in solar radiation are buffered in the lower atmosphere over land, this symbolises the strong coupling between the land surface and the lower atmosphere, with this coupling nevertheless being constrained by the energy balance over day and night over the whole surface-atmosphere system. Our approach could thus serve as the basis for a parsimonious approach to better understand land surface-atmosphere interactions.

Our interpretation that diurnal heat storage variations explain the difference in climate sensitivity can, clearly, be analysed in much greater detail in observations, reanalyses, and climate models. The variations in heat content in the lower atmosphere should be relatively straightforward to analyse in observations and model output, and one would expect a noticeable difference in variations over oceans and land. However, such an analysis requires the ability to diagnose diurnal heat storage variations in the atmosphere at sufficient temporal resolution. Measurements by radiosoundings, for instance, are available only twice a day, which is insufficient to diagnose the magnitude of diurnal heat storage variations. For climate models, this would require a substantial amount of model output at the diurnal scale, which is typically not available. It would thus require measurements and output at higher temporal resolution to evaluate these heat storage variations in greater detail.

One can also evaluate and extend this approach with respect to some of the simplifying assumptions, for instance regarding evaporation (as already discussed above), the ground heat flux, and different land cover types as well as inland water bodies such as lakes and rivers. While the role of the ground heat flux has been neglected here for the land surface, observations show a noticeable magnitude of this flux, especially for non-forested surfaces. Our interpretation would suggest that regions with a greater ground heat flux would show diurnal temperature variations that are reduced for the given radiative forcing and somewhat more similar to ocean surfaces (resulting in a lower ratio $\phi$ ). To evaluate this further and go into more regional variations in the diurnal temperature range would, however, require a more specific treatment of the different factors that vary geographically. For instance, the ground heat flux is typically larger in desert regions, which are dry, lack evaporative cooling, and typically have comparatively low optical thicknesses. To expand this approach to regional variations and different processes would thus require more spatial details in the forcing and may need to consider other relevant effects (such as evaporation and lateral heat transport), but would form interesting extensions for further research.

The ability of our rather simple formulation of the surfaceatmosphere system to explain the difference in climate sensitivity suggests that diurnal variations in temperature contain a lot of information to learn from. When our approach is extended to derive analytical solutions of the whole diurnal cycle, with possible extensions regarding the role of the ground heat flux and evaporation, one may use observed temperature variations and invert these to infer the magnitude of turbulent fluxes at the land surface, evaporation, and other aspects of the land surface energy balance. This could provide an additional means to better understand the functioning of the highly coupled and interactive yet constrained 
land-atmosphere system that complements data-driven approaches and land surface modelling.

Last, but not least, our explanation should also be applicable to the different climate sensitivity of the seasonal cycle. It is well known that winter temperatures increase more strongly than summer temperatures with global warming, particularly at high latitudes. Our interpretation here would suggest that this can be explained by winter conditions being shaped by short hours of daylight. As turbulent fluxes would only play a role during daylight in winter, this should result in a longer period of the whole day which are shaped by stable conditions and which are more sensitive to changes in longwave radiation. This longer period of stable conditions, in turn, could result in values of $\phi$ that exceed $\phi=1.5$. (Note that here we assumed an equal length of daytime and nighttime, which resulted in the amplification of $50 \%$.) In the extreme case of the polar night, one would actually expect a ratio of $\phi=2$ in the absence of any absorption of solar radiation, so stable conditions prevail over the whole day. During the polar day, one would still expect a ratio of $\phi=1.5$ because the variations in solar radiation are still buffered by the lower atmosphere, resulting in a heat gain over half of the day, while losing heat over the other half of the day. This, in turn, can explain why our value of $\phi=1.5$ appears to set a lower bound in the comparison to climate models (as shown in Fig. 6), and this can explain the greater sensitivity of high latitudes to global warming. However, a detailed analysis would be necessary in future research to substantiate this reasoning.

\section{Conclusions}

We attributed the different climate sensitivities of ocean and land surfaces to the different way by which the surfaceatmosphere system buffers the strong diurnal variations in solar radiation. This explanation was illustrated with a physically based representation of the surface energy balance in which turbulent fluxes were constrained by thermodynamics and where the two different means of buffering diurnal variations were incorporated. We then showed that our representation predicts a ratio of climate sensitivities of ocean and land surfaces that is very close to CMIP5 simulations. We furthermore showed that our interpretation also predicts a difference in the sensitivity of minimum and maximum temperatures over land that is consistent with observations. We thus conclude that the difference in climate sensitivities is primarily due to the different means by which the diurnal cycle is buffered.

Our explanation of the higher climate sensitivity of land surfaces represents this as a first-order effect that is associated with the difference in how the land-atmosphere system buffers diurnal variations compared to the ocean-atmosphere system. It demonstrates that this central aspect of global warming can be understood in simple, physical terms when using a systems approach that combines the surface with the overlying atmosphere. A quite critical implication for this explanation is that when policy deals with a global warming target expressed by a global mean temperature, it actually describes a target that is generally greater over land by about $31 \%$, as expressed by Eq. (22). For a global warming target of $2.0^{\circ} \mathrm{C}$, as is currently being discussed in climate change policy, the greater sensitivity on land implies a mean warming of $2.6^{\circ} \mathrm{C}$ over land. This difference in climate sensitivity of ocean and land would thus have important implications for society as it will experience a greater warming than formulated by a global mean warming target.

Data availability. No data were generated in this paper. CMIP5 model output was accessed through the Earth System Science Portals using the node https://esgf-data.dkrz.de/projects/esgf-dkrz/. Data download was done with the software tool synda, https:// github.com/Prodiguer/synda. 
Appendix A

Table A1. Overview of CMIP5 model output data used in the study. Version denotes the version used of the dataset as provided by the CMIP5 data portal. Abbreviations of CMIP5 models are taken from http://cmip-pcmdi.llnl.gov/cmip5/docs/CMIP5_modeling_groups.pdf.

\begin{tabular}{|c|c|c|c|c|c|}
\hline Model & Centre & Period $4 \times \mathrm{CO}_{2}$ & Period PI & Version $4 \times \mathrm{CO}_{2}$ & Version PI \\
\hline ACCESS1-0 & $\begin{array}{l}\text { Commonwealth Scientific and Industrial Research Organisation } \\
\text { and Bureau of Meteorology, Australia }\end{array}$ & $400-449$ & $750-799$ & v2 & v20120329 \\
\hline ACCESS1-3 & & $351-400$ & $700-749$ & $\mathrm{v} 1$ & $\mathrm{v} 1$ \\
\hline bcc-csm1-1 & Beijing Climate Center, China Meteorological Administration & $260-309$ & $451-500$ & v1 & $\mathrm{v} 1$ \\
\hline bcc-csm1-1-m & & 340-389 & $351-400$ & v20120910 & v20120705 \\
\hline CanESM2 & Canadian Centre for Climate Modelling and Analysis & 1950-1999 & $2961-3010$ & v20111027 & v20120623 \\
\hline CCSM4 & National Center for Atmospheric Research & $1951-2000$ & $1251-1300$ & v20120604 & v20130510 \\
\hline CNRM-CM5 & $\begin{array}{l}\text { Centre National de Recherches Meteorologiques / 1950-1999 } \\
\text { Centre Europeen de Recherche et } \\
\text { Formation Avancee en Calcul Scientifique }\end{array}$ & $2650-2699$ & v20110701 & v20110701 & \\
\hline CNRM-CM5-2 & & 1940-1989 & $2159-2208$ & v20130402 & v20130402 \\
\hline GFDL-CM3 & NOAA Geophysical Fluid Dynamics Laboratory & $96-145$ & $451-500$ & v20120227 & v20120227 \\
\hline GFDL-ESM2G & & $246-295$ & 446-495 & v20120830 & v20120830 \\
\hline GFDL-ESM2M & & $251-300$ & $446-495$ & v20130214 & v20130214 \\
\hline GISS-E2-H & NASA Goddard Institute for Space Studies & $1951-2000$ & 2900-2949 & v20160505 & v20160511 \\
\hline GISS-E2-R & & 1951-2000 & $4481-4530$ & v20160505 & v20160511 \\
\hline HadGEM2-ES & $\begin{array}{l}\text { Met Office Hadley Centre } \\
\text { (additional HadGEM2-ES realisations contributed } \\
\text { by Instituto Nacional de Pesquisas Espaciais) }\end{array}$ & 1960-2009 & $2360-2409$ & v20111129 & v20130114 \\
\hline inmem4 & Institute for Numerical Mathematics & $2190-2239$ & $2300-2349$ & v20130207 & v20130207 \\
\hline IPSL-CM5A-LR & Institut Pierre-Simon Laplace & $2060-2109$ & $2750-2799$ & v20130506 & v20130506 \\
\hline IPSL-CM5A-MR & & 1940-1989 & 2050-2099 & v20120114 & v20111119 \\
\hline IPSL-CM5B-LR & & 1960-2009 & $2080-2129$ & v20120430 & v20120114 \\
\hline MIROC-ESM & $\begin{array}{l}\text { Japan Agency for Marine-Earth Science and Technology, } \\
\text { Atmosphere and Ocean Research Institute (The University of Tokyo) } \\
\text { and National Institute for Environmental Studies }\end{array}$ & $101-150$ & $2380-2429$ & v20120710 & v20120710 \\
\hline MIROC5 & $\begin{array}{l}\text { Atmosphere and Ocean Research Institute, } \\
\text { National Institute for Environmental Studies, } \\
\text { and Japan Agency for Marine-Earth Science and Technology }\end{array}$ & $2201-2250$ & $2620-2669$ & v20120710 & v20120710 \\
\hline MPI-ESM-LR & Max Planck Institute for Meteorology & 1950-1999 & $2800-2849$ & v20120602 & v20120602 \\
\hline MPI-ESM-MR & & 1950-1999 & $2800-2849$ & v20120602 & v20120602 \\
\hline MPI-ESM-P & & 1950-1999 & $2956-3005$ & v20120602 & v20120602 \\
\hline MRI-CGCM3 & Meteorological Research Institute & $1951-2000$ & $2301-2350$ & v20120701 & v20120701 \\
\hline NorESM1-M & Norwegian Climate Centre & $101-150$ & $1151-1200$ & v20120412 & v20120412 \\
\hline
\end{tabular}




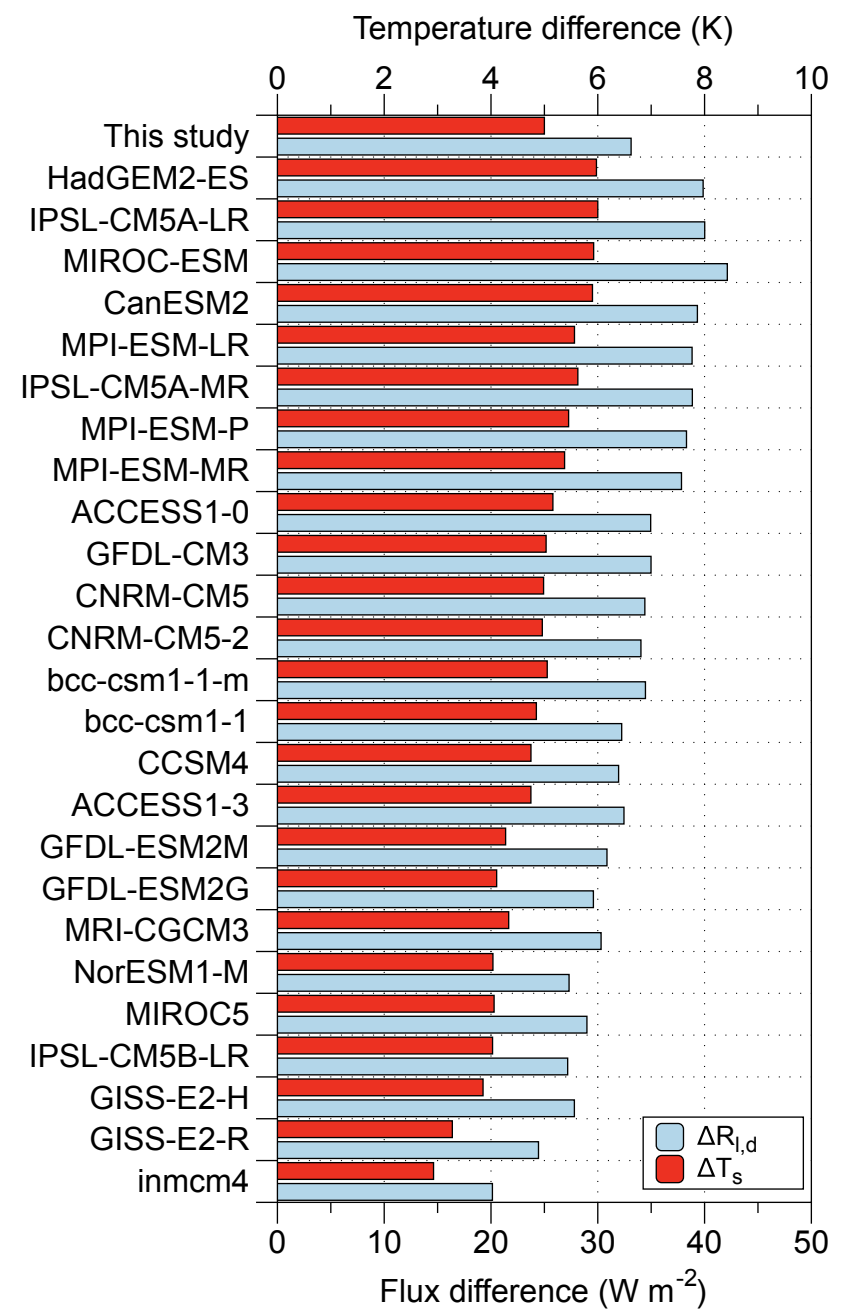

Figure A1. Global mean difference in surface temperature, $\Delta T_{\text {global }}$ (red), and the downwelling flux of longwave radiation, $\Delta R_{1, \mathrm{~d}}$ (blue), used in this study (at the top, calculated from $\Delta \tau=0.18$ ) in comparison to the equivalent change in 25 CMIP5 climate model simulations for the difference between a $4 \times \mathrm{CO}_{2}$ scenario and the preindustrial control. 
Competing interests. The authors declare that they have no conflict of interest.

Acknowledgements. We thank the two anonymous reviewers and the editor for their helpful and constructive comments. This research contributes to the "Catchments As Organized Systems (CAOS)" research group (FOR 1598), funded by the German Science Foundation (DFG). We acknowledge the World Climate Research Programme's Working Group on Coupled Modelling, which is responsible for CMIP, and we thank the climate modelling groups (listed in Table A1 of this paper) for producing and making available their model output. For CMIP the US Department of Energy's Program for Climate Model Diagnosis and Intercomparison provides coordinating support and led development of software infrastructure in partnership with the Global Organization for Earth System Science Portals.

The article processing charges for this open-access publication were covered by the Max Planck Society.

Edited by: Steven Smith

Reviewed by: two anonymous referees

\section{References}

Bennett, W. B., Wang, J., and Bras, R. L.: Estimation of Global Ground Heat Flux, J. Hydrometeorol., 9, 744-759, 2008.

Boer, G. J.: The ratio of land to ocean temperature change under global warming, Clim. Dyn., 37, 2253-2270, https://doi.org/10.1007/s00382-011-1112-3, 2011.

Bristow, K. L. and Campbell, G. S.: On the relationship between incoming solar radiation and daily maximum and minimum temperature, Agric. For. Meteor., 31, 159-166, 1984.

Budyko, M. I.: The Effect of Solar Radiation Variations on the Climate of the Earth, Tellus, 21, 611-619, 1969.

Byrne, M. P. and O'Gorman, P. A.: Land-Ocean Warming Contrast over a Wide Range of Climates: Convective Quasi-Equilibrium Theory and Idealized Simulations, J. Climate, 26, 4000-4016, https://doi.org/10.1175/JCLI-D-12-00262.1, 2013.

Easterling, D. R., Horton, B., Jones, P. D., Peterson, T. C., Karl, T. R., Parker, D. E., Salinger, M. J., Razuvayev, V., Plummer, N., Jamason, P., and Folland, C. K.: Maximum and minimum temperature trends for the globe, Science, 277, 364-367, https://doi.org/10.1126/science.277.5324.364, 1997.

Hartmann, D. L.: Global physical climatology, Academic Press, San Diego, CA, 1994.

Horton, B.: Geographical distribution of changes in maximum and minimum temperatures, Atmos. Research, 37, 101-117, 1995.

Huntingford, C. and Cox, P. M.: An analogue model to derive additional climate change scenarios from existing GCM simulations, Clim. Dynam., 16, 575-586, 2000.

Joshi, M. and Gregory, J.: Dependence of the land-sea contrast in surface climate response on the nature of the forcing, Geophys. Res. Lett., 35, L24802, https://doi.org/10.1029/2008GL036234, 2008.

Joshi, M. M., Gregory, J. M., Webb, M. J., Sexton, D. M. H., and Johns, T. C.: Mechanisms for the land/sea warming contrast ex- hibited by simulations of climate change, Clim. Dynam., 30, 455-465, https://doi.org/10.1007/s00382-007-0306-1, 2008.

Kawai, Y. and Wada, A.: Diurnal Sea Surface Temperature Variation and Its Impact on the Atmosphere and Ocean: A Review, J. Oceanogr., 63, 721-744, 2007.

Kleidon, A.: Thermodynamic foundations of the Earth system, Cambridge University Press, 2016.

Kleidon, A. and Renner, M.: Thermodynamic limits of hydrologic cycling within the Earth system: concepts, estimates and implications, Hydrol. Earth Syst. Sci., 17, 2873-2892, https://doi.org/10.5194/hess-17-2873-2013, 2013a.

Kleidon, A. and Renner, M.: A simple explanation for the sensitivity of the hydrologic cycle to surface temperature and solar radiation and its implications for global climate change, Earth Syst. Dynam., 4, 455-465, https://doi.org/10.5194/esd-4-4552013, 2013b.

Kleidon, A., Fraedrich, K., Kirk, E., and Lunkeit, F.: Maximum Entropy Production and the Strength of Boundary Layer Exchange in an Atmospheric General Circulation Model, Geophys. Res. Lett., 33, L06706, https://doi.org/10.1029/2005GL025373, 2006.

Kleidon, A., Malhi, Y., and Cox, P. M.: Maximum entropy production in environmental and ecological systems, Phil. Trans. R. Soc. B, 365, 1297-1302, 2010.

Kleidon, A., Renner, M., and Porada, P.: Estimates of the climatological land surface energy and water balance derived from maximum convective power, Hydrol. Earth Syst. Sci., 18, 2201-2218, https://doi.org/10.5194/hess-18-2201-2014, 2014.

Kleidon, A., Kravitz, B., and Renner, M.: The hydrologic sensitivity to global warming and solar geoengineering derived from thermodynamic constraints, Geophys. Res. Lett., 42, 138-144, https://doi.org/10.1002/2014GL062589, 2015.

Lambert, F. H. and Chiang, J. C. H.: Control of land-ocean temperature contrast by ocean heat uptake, Geophys. Res. Lett., 34 L13704, https://doi.org/10.1029/2007GL029755, 2007.

Lindvall, J. and Svensson, G.: The diurnal temperature range in the CMIP5 models, Clim. Dynam., 44, 405-421, https://doi.org/10.1007/s00382-014-2144-2, 2015.

Lorenz, R. D., Lunine, J. I., Withers, P. G., and McKay, C. P.: Titan, Mars and Earth: Entropy production by latitudinal heat transport, Geophys. Res. Lett., 28, 415-418, 2001.

Martyushev, L. M. and Seleznev, V. D.: Maximum entropy production principle in physics, chemistry, and biology, Phys. Reports, 426, 1-45, 2006.

Oke, T. R.: Boundary layer climates, Routledge, London and New York, second edition edn., 1987.

Ozawa, H. and Ohmura, A.: Thermodynamics of a global-mean state of the atmosphere - A state of maximum entropy increase, J. Climate, 10, 441-445, 1997.

Ozawa, H., Ohmura, A., Lorenz, R. D., and Pujol, T.: The second law of thermodynamics and the global climate system - A review of the Maximum Entropy Production principle, Rev. Geophys., 41, 1018, https://doi.org/10.1029/2002RG000113, 2003.

Paltridge, G. W.: Global dynamics and climate - a system of minimum entropy exchange, Q. J. Roy. Meteorol. Soc., 101, 475-484, 1975.

Purdy, A. J., Fisher, J. B., Goulden, M. L., and Famiglietti, J. S.: Ground heat flux: An analytical review of 6 models evaluated at 88 sites and globally, J. Geophys. Res.-Biogeosci., 121, 30453059, https://doi.org/10.1002/2016JG003591, 2016. 
Rochetin, N., Lintner, B. R., Findell, K. L., Sobel, A. H., and Gentine, P.: Radiative-convective equilibrium over a land surface, J. Climate, 27, 8611-8629, https://doi.org/10.1175/JCLI-D-13$00654.1,2014$.

Stephens, G. L., Li, J., Wild, M., Clayson, C. A., Loeb, N., Kato, S., L'Ecuyer, T., Stackhouse, P. W., Lebsock, M., and Andrews, T.: An update on Earth's energy balance in light of the latest global observations, Nature Geosci., 5, 691-696, 2012.
Sutton, R. T., Dong, B., and Gregory, J. M.: Land/sea warming ratio in response to climate change: IPCC AR4 model results and comparison with observations, Geophys. Res. Lett., 34, L02701, https://doi.org/10.1029/2006GL028164, 2007.

Taylor, K. E., Stouffer, R. J., and Meehl, G. A.: An Overview of CMIP5 and the Experiment Design, B. Am. Meteorol. Soc., 93, 485-498, 2012.

Wild, M.: Global dimming and brightening: A review, J. Geophys. Res., 114, D00D16, https://doi.org/10.1029/2008JD011470, 2009. 